\title{
Research Status of Artificial Neural Networks in Power Systems : A Review Vikash
}

\author{
Assistant Professor, Dyal Singh College, Karnal, Haryana, India
}

\begin{abstract}
ANNs in particular, have involved enormous attention due to the variety of advantages they offer over the conventional method. A standout amongst the most energizing and potentially productive ongoing developments is expanding use of Artificial Intelligence techniques. ANNs in particular, have involved enormous attention due to the variety of advantages they offer over the conventional method. Among these advantages the self-adaptive, flexible computational tool, fast speed, and robustness are the most profound. This paper presents an overview of ANNs in power system subjects like load forecasting, transient stability analysis and economic load dispatch.
\end{abstract}

Keywords : Neural Network, Load Forecasting, Economic Dispatch, Power System and Transient Stability

\section{INTRODUCTION}

Artificial intelligence is based around the idea that human intelligence can be defined in such exact terms that a machine can mimic it. The goals of artificial intelligence include learning, reasoning and perception, and machines are wired using a crossdisciplinary approach based in mathematics, computer science, linguistics, psychology and more. Three major families of AI techniques that are considered to be applied in power systems are:

- Expert Systems

- Fuzzy Logic

- Artificial Neural Networks

An Artificial Neural Network (ANN) is an information processing paradigm that is inspired by the way biological nervous systems, such as the brain, process information. The key element of this paradigm is the novel structure of the information processing system. It is composed of a large number of highly interconnected processing elements (neurons) working in unison to solve specific problems. An ANN is configured for a specific application, such as pattern recognition or data classification, through a learning process. Learning in biological systems involves adjustments to the synaptic connections that exist between the neurons. This paper is an overview of applications of ANNs in power system operation and control. It is to be noted that the following areas have attracted the most attention in the past eight years:

- Economic load dispatch

- Load forecasting

- Security assessment

- Fault Detection

- Transient stability

In this paper we will discuss the applications of ANNs in Load forecasting, Transient stability analysis, and Economic load dispatch. 


\section{ARTIFICIAL NEURAL NETWORK}

\section{A. An Artificial Neuron}

An artificial neuron is a connection point in an artificial neural network. Artificial neural networks, like the human body's biological neural network, have a layered architecture and each network node (connection point) has the capability to process input and forward output to other nodes in the network. In both artificial and biological architectures, the nodes

are called neurons and the connections are characterized by synaptic weights, which represent the significance of the connection. As new data is received and processed the synaptic weights change and this is how learning occurs. An example of an artificial neuron is shown in Fig. 1.

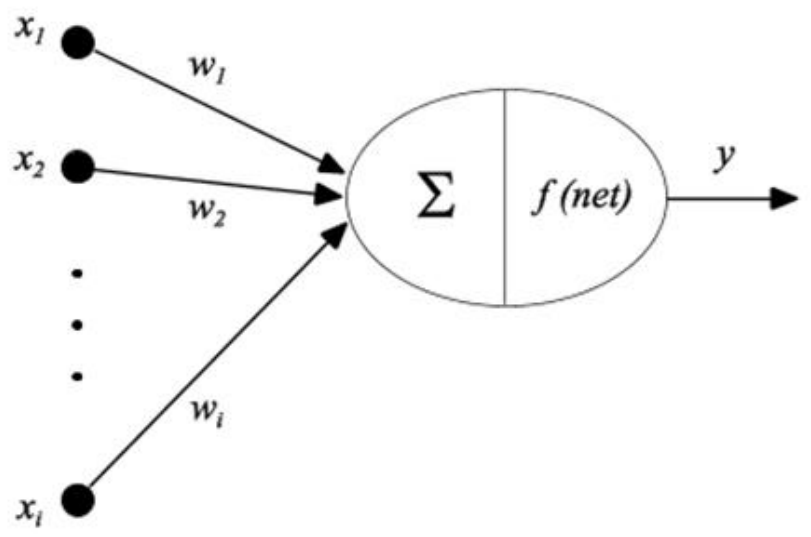

Figure 1. An Artificial Neuron.

\section{B. ARCHITECTURE OF NEURAL NETWORK}

Networks are formed from trillions of neurons (nerve cells) exchanging brief electrical pulses called action potentials. Computer algorithms that mimic these biological structures are formally called artificial neural networks to distinguish them from animals. Neural network research is motivated by two desires: to obtain a better understanding of the human brain, and to develop computers that can deal with abstract and poorly defined problems. For example, conventional computers have trouble understanding speech and recognizing people's faces. In comparison, humans do extremely well at these tasks.

Many different neural network structures have been tried, some based on imitating what a biologist sees under the microscope, some based on a more mathematical analysis of the problem. This neural network is formed in three layers, called the input layer, hidden layer, and output layer. Each layer consists of one or more nodes, represented in this diagram by the small circles. The lines between the nodes indicate the flow of information from one node to the next. In this particular type of neural network, the information flows only from the input to the output (that is, from left-to-right). Other types of neural networks have more intricate connections, such as feedback paths.

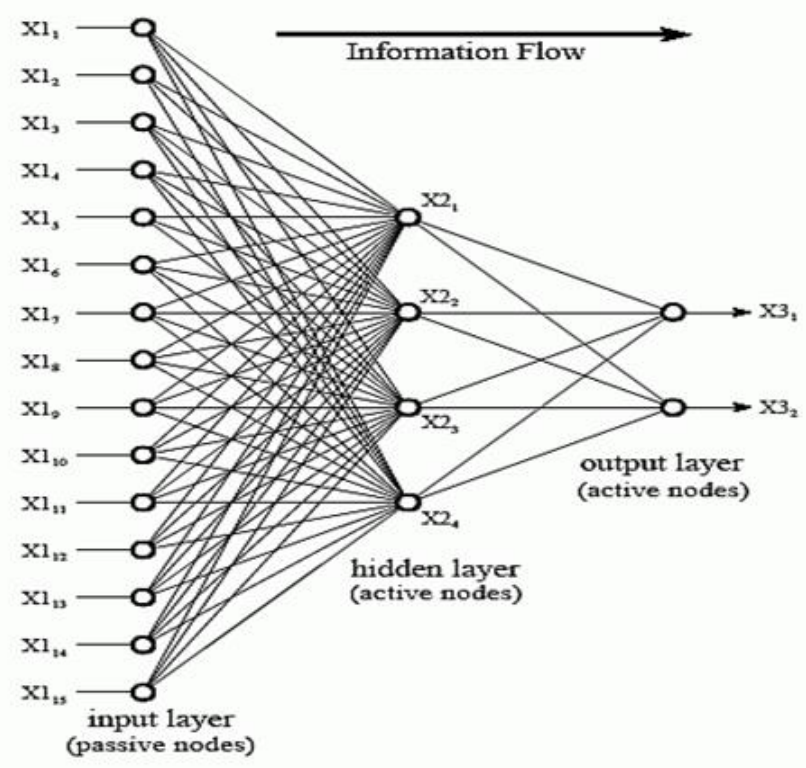

Figure 2. Architecture of a Neural Network.

The nodes of the input layer are passive, meaning they do not modify the data. They receive a single value on their input, and duplicate the value to their multiple outputs. In comparison, the nodes of the hidden and output layer are active. This means they modify the data. The variables: X11X12...X115 holds the data to be evaluated. Each value from the input 
layer is duplicated and sent to all of the hidden nodes. This is called a fully interconnected structure. The values entering a hidden node are multiplied by weights, a set of predetermined numbers stored in the program. The weighted inputs are then added to produce a single number. This basic architecture of neural network with one hidden layer is shown in Fig 2. Before leaving the node, this number is passed through a nonlinear mathematical function called a sigmoid. This is an "s" shaped curve that limits the node's output. That is, the input to the sigmoid is a value between $-\infty$ and $+\infty$, while its output can only be between 0 and 1 . The outputs from the hidden layer are represented in the flow diagram by the variables: X21,X22,X23 and X24. Just as before, each of these values is duplicated and applied to the next layer. The active nodes of the output layer combine and modify the data to produce the two output values of this network, X31 and X32.Neural networks can have any number of layers, and any number of nodes per layer. Most applications use the three layer structure with a maximum of a few hundred input nodes. The hidden layer is usually about $10 \%$ the size of the input layer. In the case of target detection, the output layer only needs a single node. The output of this node is thresholded to provide a positive or negative indication of the target's presence or absence in the input data.

Depending upon then training Imparted, ANN can be classified as supervised ANN or unsupervised ANN. The supervised ANN requires the sets of inputs and the outputs for its training. During the training, the output from the ANN is compared with the desired output (target) and the difference (error) is reduced by employing some training algorithm. This training is repeated till the actual output acquires an acceptable level. Supervised ANN may be a feed forward or non-recurrent network such as Multi Layer Perceptron (MLP), Functional Link Net (FLN). On the other hand, the artificial neural network which does not require a supervisor or teacher for training is known as unsupervised ANN. In competitive or unsupervised learning units of the output layer compete for the chance to respond to a given input pattern. Kohonen's Self-Organizing Feature Map (SOFM) and Adaptive Resonance Theory (ART) are examples of unsupervised learning.

\section{APPLICATIONS OF NEURAL NETWORKS IN POWER SYSTEMS}

This section aids with typical ANN application areas in power systems. The various areas are: - Security assessment, transient stability, fault Detection and diagnosis, control analysis, protection and design. Most popular problems are: (i) load Forecasting (ii) transient stability analysis and (iii) Economic load dispatch. Fig 3 shows the distribution of use of ANN in the various areas of power systems.

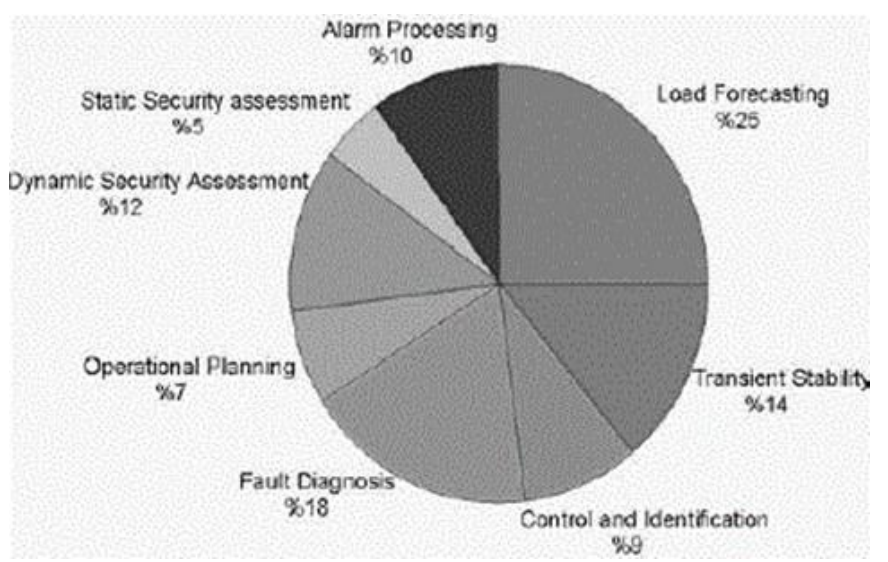

Figure3. Distribution on the basis of use of ANN.

\section{A. LOAD FORECASTING}

Electric load forecasting is the process used to forecast future electric load, given historical load and weather information and current and forecasted weather information. In the past few decades, several models have been developed to forecast electric load more accurately. Load forecasting can be divided into three major categories: Long-term electric load forecasting, used to supply electric utility company management with prediction of future needs for 
expansion, equipment purchases, or staff hiring. Medium-term forecasting, used for the purpose of scheduling fuel supplies and unit maintenance. Shortterm forecasting, used to supply necessary information for the system management of day-today operations and unit commitment.

In applying a neural network to electric load forecasting, we select one of a number of architectures (e.g., Hopfield, back propagation, Boltzmann machine), the number and connectivity of layers and elements, use of bi-directional or unidirectional links, and the number format (e.g., binary or continuous) to be used by inputs and outputs, and internally. The most popular artificial neural network architecture for electric load forecasting is back propagation. Back propagation neural networks use continuously valued functions and supervised learning. That is, under supervised learning, the actual numerical weights assigned to element inputs are determined by matching historical data (such as time and weather) to desired outputs (such as historical electric loads) in a pre-operational 'training session'.

\section{B. TRANSIENT STABILITY ANALYSIS}

Transient stability is the ability of the system to stay in synchronism when subjected to a large disturbance. Due to large disturbances like faults, large load variations, generator outages etc there will be large swings in the electrical power outputs, rotor angles, rotor speeds and bus voltages of the generators in the system. Due to this reason a power system cannot be linearized, like in the case of small signal stability analysis. The system cannot be linearized for large disturbances, hence Eigen value analysis cannot be used for assessing the stability as the system is nonlinear. One way of assessing the effect of large disturbances on the system stability is through numerical solution of differential algebraic equations (DAE). The time of interest for transient stability assessment is typically 2 to 10 seconds after a disturbance. During this time usually it is assumed that the turbine and turbine governors will not be acting as they are mechanical systems. Hence, for transient stability analysis usually the turbine and speed governor dynamics are not considered and the input mechanical torque TM is considered constant.

Many researchers have been working on the use of artificial neural networks in transient stability analysis.

The Critical Clearing Time (CCT) is a very important parameter in order to maintain the stability of power systems. The CCT is the maximum time duration that a fault may occur in power systems without failure in the system so as to recover to a steady state operation. Some works have been carried out to determine the CCT of power systems using the feed forward multilayer perceptron (MLP) with back propagation learning. ANN method based on fuzzy ARTMAP architecture is also used to analyze TSA of a power system. Works have also been published for determining the transient stability using Probabilistic Neural Networks.

\section{ECONOMIC LOAD DISPATCH}

The economic load dispatch problem can be defined as determining the least-cost power generation schedule from a set of on-line generating units to meet the total power demand at a given point of time. The generating unit cost and the network transmission losses are modeled as quadratic function of the power generation.

Several methods have been used in past for solving economic dispatch problems including Lagrangian relaxation method, linear programming(LP) techniques specially dynamic programming(DP), Beale's quadratic programming, Newton-Raphson's economic method, Lagrangian augmented function, 
and recently Genetic algorithms and NNs. But, main drawbacks of genetic algorithm for ED are difficult to define the fitness function, find the several suboptimum solutions without guaranty that this solution isn't locally and longer search time. Neural networks and specially the Hopfield model, have a well-demonstrated capability of solving combinational optimization problem. This model can be used to solve the conventional ELD problems for units with continuous quadratic, piecewise quadratic, or cubic fuel cost functions.

\section{CONCLUSION}

This paper gives a brief introduction about the Artificial Neural Network approach of Artificial Intelligence. ANN is nonlinear model that is easy to use and understand compared to statistical methods. ANN is non-parametric model while most of statistical methods are parametric model that need higher background of statistic. ANN with Back propagation (BP) learning algorithm is widely used in solving various classification and forecasting problems. Even though BP convergence is slow but it is guaranteed. However, ANN is black box learning approach, cannot interpret relationship between input and output and cannot deal with uncertainties. To overcome this several approaches have been combined with ANN such as feature selection and etc.

This paper further reviews the various applications of AI to power systems putting particular emphasis on load forecasting, economic load dispatch and transient stability analysis. The present day prime difficulties and their proposed solutions are reviewed finally; the integration of ANNs with other emerging technologies such as FS, GA etc. was discussed as a future research direction.

\section{REFERENCES}

[1]. H.Singh, M.S. Sachdev, T.S. Sidhu "Design, Implementation and Testing of an Arti_cial Neural Network Based Fault Direction Discriminator for protecting Transmission Lines," IEEE Transactions on Power Delivery

[2]. H. S. Hippert, C.E. Pedreira, R.C. Souza, Februrary (2001) Neural Networks for Short- Term Load Forecasting: A Review and Evaluation, IEEE Trans. on Power System

[3]. K.W. Chan, A.R. Edward, A.R. Danish, (2002) OnLine Dynamic Security Contingency Screening Using Artificial Neural Network, IEEE Trans. on Power Distribution System

[4]. A.K. Sinha, Short Term Load Forecasting Using Artificial Neural Networks, IEEE Trans. On Power System Distribution

[5]. N. Kumarappan, M.R. Mohan, S. Murugappa, ANN Approach to Combined Economic and Emission Dispatch for Large-Scale System, IEEE Power Distribution system

[6]. D. Niebur and T.S. Dillon, (1996) Neural Network Applications in Power Systems CRL Publishing Ltd. U.K.

[7]. Sadat Hadi, (1997) Power System Analyses Mc Graw Hill Publication, New Delhi.

[8]. Malik, N., April (2005) Artificial Neural Network and Their Application, Proc. of National Conference on Unearthing Technological developments \& Their Transfer for Serving Masses, Mathura, India.

[9]. Pothisarn, C. and S. Jiriwibhakorn, 2003, Critical clearing time determination of EGAT system using artificial neural networks. IEEE Power Engineering Society General Meeting.

[10]. Sanyal, K. K., 2004, Transient stability assessment using neural network. IEEE International Conference on Electric Utility Deregulation, Restructuring and Power Technologies

\section{Cite this article as :}

Vikash, "Research Status of Artificial Neural Networks in Power Systems : A Review", International Journal of Scientific Research in Computer Science, Engineering and Information Technology (IJSRCSEIT), ISSN : 2456-3307, Volume 5, Issue 3, pp.664-668, May-June-2019. Available at doi : https://doi.org/10.32628/CSEIT1952202 Journal URL : https://ijsrcseit.com/CSEIT1952202 